\title{
Cooperative transmission scheme to address random orientation and blockage events in VLC systems
}

\author{
Borja Genovés Guzmán ${ }^{1}$, Alexis A. Dowhuszko ${ }^{2}$, Víctor P. Gil Jiménez ${ }^{1}$ and Ana I. Pérez-Neira ${ }^{2,3}$. \\ Email: bgenoves@tsc.uc3m.es, alexis.dowhuszko@cttc.es, vgil@tsc.uc3m.es, ana.perez@cttc.es \\ ${ }^{1}$ Dept. of Signal Theory and Communications, Universidad Carlos III de Madrid (UC3M), Madrid, Spain. \\ ${ }^{2}$ Centre Tecnològic de Telecomunicacions de Catalunya (CTTC/CERCA), Barcelona, Spain. \\ ${ }^{3}$ Dept. of Signal Theory and Communications, Universitat Politècnica de Catalunya (UPC), Barcelona, Spain.
}

\begin{abstract}
Visible light communication (VLC) is a promising communication technology that paves the way to the Internet of Things. The forthcoming Industry 4.0 requires reliable high-speed communication links, which can be easily fulfilled with the massive adoption of the VLC technology. However, in VLC indoor communication environments, line-of-sight link blockages and strong misalignment losses between the lightemitting diode (LED) transmitters and the photodiode (PD) receivers are usual problems that must be tackled. This paper proposes a coordinated multi-point (CoMP) transmission technique to deal with both issues, and carries out a detailed evaluation of the VLC system performance as function of the PD receiver orientation and the density of obstacles in different scenarios. The proposed cooperative transmission scheme demonstrates an improvement in the average cell data rate and outage probability performance and, as a consequence, justifies the use of CoMP techniques to provide reliable VLC services in ultra-dense small cell scenarios indoors.
\end{abstract}

\section{INTRODUCTION}

Visible light communication (VLC) is an emerging technology that allows communication and illumination services simultaneously [1]. VLC can complement the overwhelmed radiofrequency $(\mathrm{RF})$ communication systems that are constantly struggling to cope with the increasing demand of wireless data services. Thus, VLC is a suitable solution to comply with the requirements of contemporary wireless communication services. VLC takes advantage of the high modulation speed of the light-emitting diode (LED) luminaries to transmit information through the fast variation of the light intensity. Intensity modulation (IM) is used in the LED transmitters, whereas direct detection (DD) is carried out by photodiodes (PDs) performing as receivers. Thanks to the benefits that orthogonal frequency division multiplexing (OFDM) schemes provide, much effort has been dedicated to adapt the traditional OFDM waveform designs into optical-OFDM (O-OFDM) ones, giving rise to direct-current biased optical OFDM (DCO-OFDM) and asymmetrically clipped optical OFDM (ACO-OFDM) schemes [2], among others. However, the need of a line-of-sight (LoS) link between the transmitter and the receiver, as well as the high optical inter-cell interference that is produced in multi-cell scenarios, has delayed the massive adoption of the VLC technology so far.

In accordance with the current Industry 4.0 trend, which is characterized by a great flexibility and mobility of wireless-enabled terminals, a multi-cell VLC system can provide a seamless coverage by deploying LED luminaries in a scenario where every place needs to be well illuminated. However, the notable overlap that exists between coverage areas of adjacent LED lamps can produce a high inter-cell interference level. Traditionally, fractional frequency reuse
(FFR) and coordinated multi-point (CoMP) techniques, both of them included in different releases of the 3GPP standardization [3], have been employed to minimize the impact of the inter-cell interference problem.

In a VLC system, the presence of a LoS link between the LED and the PD is essential to provide a suitable data rate performance. In practice, two phenomena can prevent the existence of a LoS condition in a VLC system, namely: (a) The presence of opaque obstacles in the room; (b) The random orientation that the PD can take, generating a misalignment between the transmitter and receiver. In this context, CoMP represents an efficient technique to combat both kinds of impairments. In VLC, CoMP was proposed in [4] to enable handover and mobility within a room, and in [5] to improve the data rate performance when using dynamic subcarrier allocation and access point (AP) selection. Similarly, spatial division multiple access (SDMA) techniques were proposed in [6] to serve, from multiple APs, a group of users that had been previously clustered. So far, most of the CoMP transmission techniques that have been proposed in the literature for VLC were dynamic and dependent on the instantaneous location of the users, providing uncertainty and introducing extra complexity in the system design. To address this limitation, static CoMP techniques were presented in [7] and [8] for different modulation schemes, in which time division multiple access (TDMA) was utilized to provide orthogonal resources among neighboring cells due to the lack of independent dimensions to enable the simultaneous transmission in adjacent APs.

In this work, red-green-blue (RGB) LEDs and wavelength division multiplexing (WDM) [9] are utilized to increase the communication dimensions and flexibility of the VLC system, avoiding the use of TDMA that decreases the mean cell data rate performance considerably. The orientation of the receiver is evaluated when cooperative and non-cooperative transmission schemes are used. Several works have already studied the effect of the receiver orientation in a singlecell VLC system without multi-point cooperation [10] [11]. However, this paper extends the analysis to a static cooperative transmission scheme, evaluating the impact of the random orientation of the PD in presence of different working conditions with LoS blockage.

The structure of this work is organized as follows. Section II introduces the system model and explains the cooperative and non-cooperative transmission schemes that are analyzed. Section III presents the simulation results and analyzes the effect of random orientation and LoS blockage events in terms of the average cell data rate and the outage probability. Finally, Section IV draws the conclusions. 


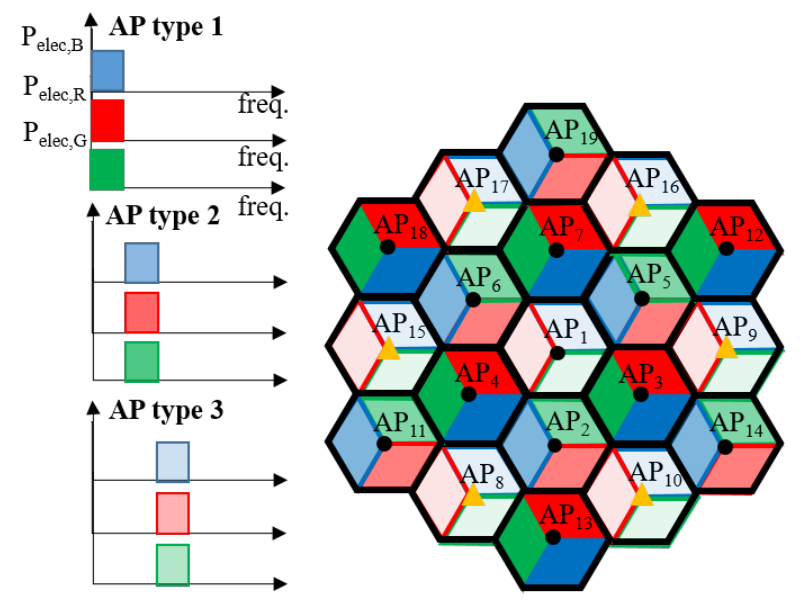

(a) Non-cooperative scheme
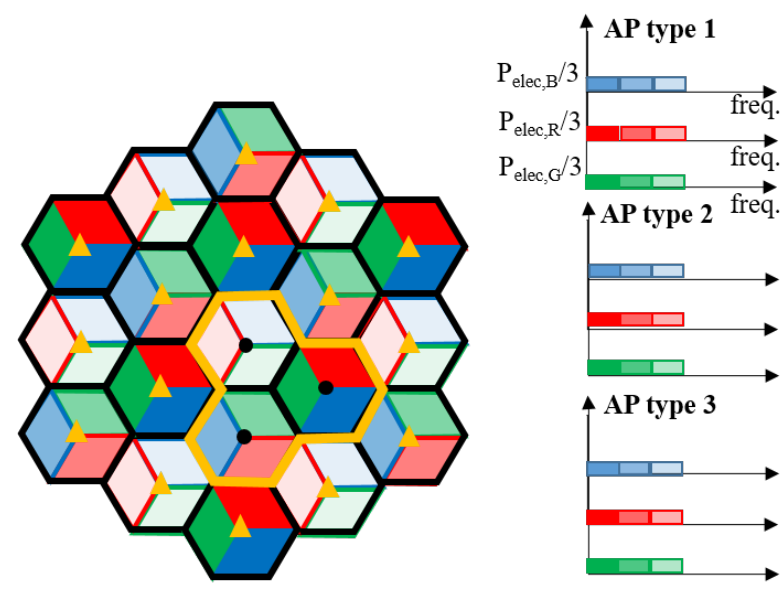

(b) Cooperative scheme. Solid yellow line: Cooperation area.

Fig. 1: Two-tier multi-cell VLC network with $|\mathcal{S}|=3$ sectors (per cell) and $|\mathcal{F}|=3$ electrical frequency bands (per chip color). The power allocation profile is also represented for the different AP types. The APs that interfere with the reference green sector of $\mathrm{AP}_{1}$ are represented with solid yellow triangles.

\section{SYSTEM MODEL}

Figure 1 illustrates the system model that is used in this paper, which consists of a two-tier multi-cell scenario with 19 APs with index $i \in\{1, \ldots, 19\}$. Every AP transmits information using WDM with RGB LEDs, sending three independent data streams simultaneously. Every LED color chip serves a sector in the reference cell and, when cooperation is implemented (see Fig. 1b), every LED color chip serves in addition two more sectors of neighboring cells; therefore, in case of cooperation, each tri-color LED transmits information to nine cell sectors in total. Depending on its location, each user will demodulate the data signal that is transmitted on a specific color and frequency sub-band. The index of the color chip is denoted with the variable $c \in\{\mathrm{R}, \mathrm{G}, \mathrm{B}\}$, and every color chip transmits the same electrical power (i.e., $P_{\text {elec,c }}$ ) to provide an objective white color for illumination. To make a fair comparison, when cooperation is carried out, this electrical power is equally divided into the three electrical frequency sub-bands of each chip color. Thus, only a third part of the power (i.e., $P_{\text {elec,c }} / 3$ ) is allocated in each sub-band of each color when implementing the cooperation scheme in transmission. In Fig. 1, the AP locations are represented with solid black circles and are arranged following a hexagonal cell pattern. The green sector of AP with index $i=1$ is used as reference sector for evaluation purposes, in which case the interfering APs locations are highlighted with yellow triangles in Fig. 1. Thanks to the hexagonal cell pattern and the proposed resource allocation, the study of the data rate performance in this reference sector will be representative for the whole multi-cell scenario. The number of sectors per cell and frequency sub-bands per color chip are denoted by $|\mathcal{S}|$ and $|\mathcal{F}|$, respectively.

The input signal in the LED color chip $c$ must be real and non-negative. Thus, the vector of symbols that is fed into the inverse fast Fourier transform (IFFT) block must satisfy the Hermitian symmetry property, i.e.,

$$
\mathbf{X}_{\mathrm{H}, c}=\left[\begin{array}{llll}
0 & \left\{X_{c}[k]\right\}_{k=1}^{K / 2-1} & 0 & \left\{X_{c}[K / 2-k]^{*}\right\}_{k=1}^{K / 2-1}
\end{array}\right], c \in \mathcal{C},(1)
$$

where the symbols in the 0 -th and $K / 2$-th subcarriers must be zero-valued. The frequency-domain symbol in the $k$-th subcarrier transmitted by the color chip $c$ is denoted by $X_{c}[k]$. If DCO-OFDM is employed, a DC-bias $I_{\mathrm{dc}, c}$ must be added to the time-domain signal to get a non-negative signal that attains the form

$$
x_{\mathrm{tx}, c}(t)=x_{c}^{\prime}(t)+I_{\mathrm{dc}, c}, \quad c \in \mathcal{C} .
$$

In the previous equation, the time-domain signal $x_{c}^{\prime}(t)$ is obtained after scaling and clipping the signal at the output of the IFFT block, which is denoted by $x_{c}(t)$. Taking into account the dynamic range of the LED in which the transfer function can be approximated as linear, we define

$$
x_{c}^{\prime}(t)=\left\{\begin{array}{lc}
\lambda_{\mathrm{t}} & : \lambda_{\mathrm{t}}<\sqrt{P_{\text {elect }, c} / \nu} \cdot x_{c}(t) \\
\sqrt{P_{\text {elect }, c} / \nu} \cdot x_{c}(t) & : \lambda_{\mathrm{b}} \leq \sqrt{P_{\text {elect }, c} / \nu} \cdot x_{c}(t) \leq \lambda_{\mathrm{t}} \\
\lambda_{\mathrm{b}} & : \sqrt{P_{\text {elect }, c} / \nu} \cdot x_{c}(t)<\lambda_{\mathrm{b}}
\end{array}\right.
$$

where $\sqrt{P_{\text {elect }, c} / \nu}$ is the scaling factor for color $c$, in which $\nu=1$ or $\nu=3$ in case of implementing a non-cooperative or cooperative transmission scheme, respectively. Note that $\nu$ can also be interpreted as the number of sub-bands that are used per color chip and AP (see Fig. 1). The top and bottom clipping levels are denoted by $\lambda_{\mathrm{t}}$ and $\lambda_{\mathrm{b}}$, respectively. The frequency domain symbol demodulated at the $k$-th subcarrier by a generic user can be formulated as

$$
\begin{aligned}
Y[k]= & \eta_{\mathrm{pd}} \eta_{\text {led }, c}\left(\sum_{i \in \mathcal{D}} X_{i, c}^{\prime}[k] H_{i}+\sum_{i \in \mathcal{I}} X_{i, c}^{\prime}[k] H_{i}\right)+N_{\mathrm{rx}, c}[k], \\
& c \in \mathcal{C},
\end{aligned}
$$

where $\eta_{\text {led,c }}$ is the electrical to optical conversion efficiency for the corresponding LED color chip, $\eta_{\mathrm{pd}}$ is the responsivity of the PD, and $\mathcal{D}(\mathcal{I})$ contains the indices of the cooperating (interfering) APs. The frequency-domain symbol transmitted in the $k$-th subcarrier of LED chip $c$ is denoted by $X_{i, c}^{\prime}[k]$, whereas $N_{\mathrm{rx}, c}[k]$ is the frequency-domain noise in reception on the $k$-th subcarrier of color $c$, which follows a Gaussian distribution with variance

$$
\sigma_{\mathrm{rx}, c}^{2}=\frac{N_{0, c} W}{|\mathcal{F}| \xi^{2}}, \quad c \in \mathcal{C},
$$

where $W$ is the aggregate bandwidth of the $|\mathcal{F}|$ frequency sub-bands of the LED chip color, and $\xi=\sqrt{K /(K-2)}$ is the factor that scales the power accordingly among the active 


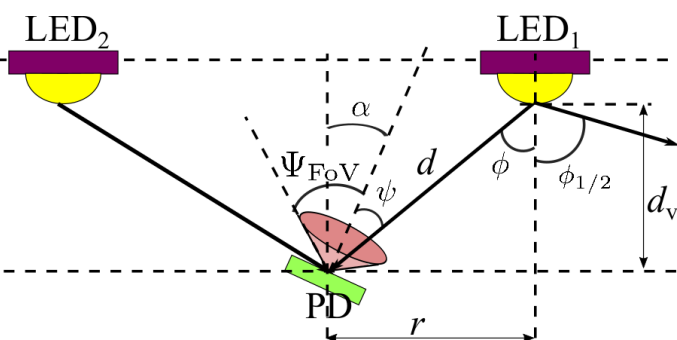

Fig. 2: Geometry of a VLC link in which the field-of-view cone of the PD contains (does not contain) the LoS link from the LED $\left(\mathrm{LED}_{2}\right)$. Therefore, the PD is classified as aligned (misaligned) with the $\mathrm{LED}_{1}\left(\mathrm{LED}_{2}\right)$.

DCO-OFDM subcarriers. The power spectral density of noise at the LED chip $c$ is formulated by

$$
\begin{aligned}
N_{0, c} & =N_{0, \mathrm{~s}, c}+N_{0, \mathrm{th}} \\
& =2 q \eta_{\mathrm{pd}}\left(\sum_{i} P_{\mathrm{opt}, c} H_{i}+E_{\mathrm{r}, \mathrm{ab}} A_{\mathrm{pd}}\right)+\frac{4 \kappa_{\mathrm{B}} T_{\mathrm{abs}}}{R_{\mathrm{L}}}, c \in \mathcal{C},(6)
\end{aligned}
$$

where $N_{0, \mathrm{~s}, c}$ and $N_{0, \text { th }}$ are power spectral densities of the shot and thermal noise components, respectively. In the latter formula, $q$ represents the charge of the electron, $\sum_{i} P_{\mathrm{opt}, c} H_{i}$ is the received optical power in color $c$ that comes from the LEDs, whereas $E_{\mathrm{r}, \mathrm{ab}} A_{\mathrm{pd}}$ denotes the optical power that comes from the ambient light $\left(E_{\mathrm{r}, \mathrm{ab}}\right.$ is the incident irradiance and $A_{\mathrm{pd}}$ is the physical area of the PD). The thermal noise spectral density is represented as a function of the Boltzmann constant $\kappa_{\mathrm{B}}$, the absolute temperature $T_{\mathrm{abs}}$, and the load resistance $R_{\mathrm{L}}$ of the receiver. Finally,

$$
H_{i}=\frac{(m+1) A_{\mathrm{pd}}}{2 \pi d_{i}^{2}} \cos ^{m}\left(\phi_{i}\right) \cos \left(\psi_{i}\right) \mathbb{I}_{\text {align }, i}\left(\psi_{i}\right) \mathbb{I}_{\text {block }, \mathrm{i}},
$$

is the optical wireless channel gain between AP $i$ and the user under study, where $d_{i}=\sqrt{r_{i}^{2}+d_{\mathrm{v}}^{2}}$ is the Euclidean distance that depends on the horizontal distance $r_{i}$ and vertical distance $d_{\mathrm{v}}$. For the sake of clarification, all the parameters of this geometrical setting are represented in Fig. 2. The angle of emission and the angle of incidence between the $\mathrm{AP}_{i}$ and the PD are denoted by $\phi_{i}$ and $\psi_{i}$, respectively. The indicator function $\mathbb{I}_{\text {align }, i}\left(\psi_{i}\right)$ equals $1(0)$ if the PD is aligned (not aligned) with the $i$-th AP in such a way that the LoS path falls inside (outside) the field-of-view (FoV) $\Psi_{\text {FoV }}$. Similarly,

$$
\mathbb{I}_{\text {block }, \mathrm{i}}= \begin{cases}1 & \text { if no obstacle between } \mathrm{PD} \text { and } \mathrm{AP}_{i} \\ 0 & \text { if obstacle between } \mathrm{PD} \text { and } \mathrm{AP}_{i}\end{cases}
$$

The Lambertian index of the transmitter is computed by

$$
m=-1 / \log _{2}\left(\cos \left(\phi_{1 / 2}\right)\right),
$$

where $\phi_{1 / 2}$ is the half-power semi-angle of the LED. Finally, a cyclic prefix is assumed on the OFDM signal, whose duration is longer than the channel delay spread in order to avoid the effect of multipath. Although reflections could be used to collect power in case of LoS-link blockage, this work addresses this phenomenon by means of CoMP techniques.

From (2), it is possible to see that the optical transmit power per color attains the form

$$
\begin{aligned}
P_{\mathrm{opt}, c}=\mathrm{E}\left\{\eta_{\mathrm{led}, c} \cdot x_{\mathrm{tx}, c}(t)\right\} & =\eta_{\mathrm{led}, c}\left(\mathrm{E}\left\{x_{c}^{\prime}(t)\right\}+I_{\mathrm{dc}, c}\right) \\
& \approx \eta_{\mathrm{led}, c} I_{\mathrm{dc}, c} \quad c \in \mathcal{C},
\end{aligned}
$$

\begin{tabular}{|c|c|c|c|}
\hline Parameter & Description & Value & Unit \\
\hline$R$ & Circular cell radius & 1.5 & $\mathrm{~m}$ \\
\hline$d_{\mathrm{v}}$ & Vertical separation AP-PD & 2.25 & $\mathrm{~m}$ \\
\hline$P_{\mathrm{opt}}$ & Transmitted optical power & 14 & $\mathrm{~W}$ \\
\hline$\phi_{1 / 2}$ & Half-power semi-angle & 70 & deg. \\
\hline$W$ & LED chip modulation bandwidth & 50 & $\mathrm{MHz}$ \\
\hline$K$ & Number of subcarriers & 512 & \\
\hline$\eta_{\mathrm{pd}}$ & Avalanche PD responsivity & 16 & $\mathrm{~A} / \mathrm{W}$ \\
\hline$A_{\mathrm{pd}}$ & Avalanche PD physical area & 3.14 & $\mathrm{~mm}^{2}$ \\
\hline$\Psi_{\mathrm{FoV}}$ & Receiver field of view angle & $5-90$ & deg. \\
\hline$\sigma_{\alpha}$ & Variance of the inclination angle & 45 & deg. \\
\hline$\zeta$ & $\begin{array}{l}\text { Ratio of DC-bias level to } \\
\text { standard deviation of AC signal }\end{array}$ & 5.05 & $\mathrm{~dB}$ \\
\hline$E_{\mathrm{v}, \mathrm{ab}}$ & Illuminance from ambient light & 100 & $\operatorname{lux}$ \\
\hline$R_{\mathrm{L}}$ & Receiver load resistance & 500 & $\Omega$ \\
\hline$T_{\mathrm{abs}}$ & Absolute temperature & 300 & $\mathrm{~K}$ \\
\hline
\end{tabular}

where $\mathrm{E}\{\cdot\}$ is the statistical expectation. Let us define $\zeta_{c}=$ $\frac{I_{\mathrm{dc}, c}}{\sqrt{P_{\text {elect }, c} / \nu}}$ as the ratio of DC-bias level to the standard
TABLE I: System Parameters

deviation of the transmitted electrical signal [12]; then, the electrical transmit power per color can be re-written as

$$
\frac{P_{\text {elect }, c}}{\nu}=\frac{P_{\text {opt }, c}^{2}}{\eta_{\text {led }, c}^{2} \zeta_{c}^{2}}, \quad c \in \mathcal{C} .
$$

Assuming that the optical power is evenly allocated among LED chip colors to get a white color light from the human's eye perspective, and that the same $\zeta_{c}$ is utilized per color, (11) can be generalized as

$$
\frac{P_{\text {elect }, c}}{\nu}=\frac{P_{\text {elect }}}{\nu}=\left(\frac{P_{\text {opt }}}{|\mathcal{C}| \eta_{\text {led }} \zeta}\right)^{2}, \quad c \in \mathcal{C} .
$$

The SINR, which is a key metric to evaluate the performance of a communication system, can be computed as

$$
\begin{aligned}
\Gamma\left(r_{0}, \theta\right) & =\frac{\sum_{i \in \mathcal{D}} D_{i}\left(r_{0}, \theta\right)}{\sum_{j \in \mathcal{I}} I_{j}\left(r_{0}, \theta\right)+\sigma_{\mathrm{rx}}^{2}} \\
& =\frac{\frac{1}{\nu} \eta_{\mathrm{pd}}^{2} \eta_{\text {led }}^{2} P_{\text {elect }} \xi^{2}\left|\sum_{i \in \mathcal{D}} H_{i}\left(r_{0}, \theta\right)\right|^{2}}{\frac{1}{\nu} \eta_{\mathrm{pd}}^{2} \eta_{\mathrm{led}}^{2} P_{\text {elect }} \xi^{2} \sum_{j \in \mathcal{I}}\left|H_{j}\left(r_{0}, \theta\right)\right|^{2}+\sigma_{\mathrm{rx}}^{2}\left(r_{0}, \theta\right)},
\end{aligned}
$$

where $\left(r_{0}, \theta\right)$ is the user location in polar coordinates, and $D_{i}\left(r_{0}, \theta\right)$ and $I_{j}\left(r_{0}, \theta\right)$ are the received power from the $i$-th cooperating and the $j$-th interfering APs, respectively.

The average cell data rate can be formulated as

$$
\bar{R}_{\text {cell }} \approx \frac{|\mathcal{S}| \cdot W}{2 \cdot|\mathcal{F}|} \cdot \mathrm{E}\left\{\log _{2}\left(1+\Gamma\left(r_{0}, \theta\right)\right)\right\},
$$

where the factor 2 in the denominator is due to the spectral efficiency loss when the Hermitian symmetry requirement in (1) is forced in transmission.

A new random variable (RV) $X_{i}$ that denotes the existence of a LoS-link blockage event between the AP $i$ and the PD is defined, which can be approximated as a Bernoulli $\mathrm{RV}$ distribution $X_{i} \sim \operatorname{Be}\left(\rho_{\mathrm{block}, i}\right)$, where $\rho_{\text {block }, i}$ is the probability of LoS-link blockage. Thus, $X_{i}$ takes value 1 (0) with a probability of $\rho_{\mathrm{block}, i}\left(1-\rho_{\mathrm{block}, i}\right)$. When the reference sector is studied (i.e., the green sector of $\mathrm{AP}_{1}$ ), the outage probability when the non-cooperative transmission scheme is used becomes

$$
P_{\text {out }, \text { ncoop }}=\mathrm{E}\left\{X_{1}\right\}=\rho_{\text {block }, 1} .
$$

On the other hand, in the case of cooperative scheme, the outage probability can be estimated as

$$
P_{\text {out }, \text { coop }}=\mathrm{E}\left\{X_{1} \cdot X_{2} \cdot X_{3}\right\} \approx \prod_{i=1,2,3} \rho_{\text {block }, i},
$$




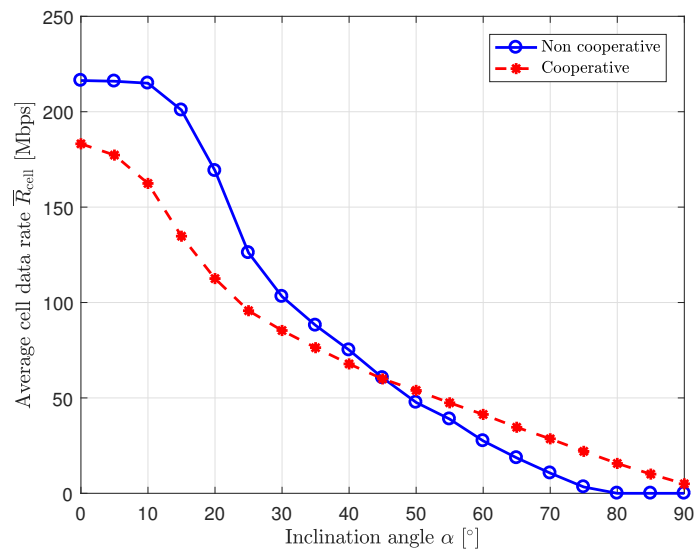

Fig. 3: Average cell data rate as a function of the inclination angle $\alpha$ for the (non-)cooperative transmission schemes with $\Psi_{\mathrm{FoV}}=45^{\circ}$.

where the latter approximation would be equality if the three distributions of RVs $X_{1}, X_{2}$ and $X_{3}$ were independent. In practice, it is possible to show that the correlation coefficient between $\mathrm{RVs} X_{i}$ varies according to the location of the PD in the inner/outer part of the serving cell.

\section{Simulation Results}

This section compares the average cell data rates and the outage probability when cooperative and non-cooperative transmission schemes are used. The system parameters are shown in Table I. Regarding the orientation of the PD, unless otherwise specified, the inclination angle of the vector that is normal to the sensitive area of the PD obeys a truncated Gaussian distribution with mean 0 , variance $\sigma_{\alpha}$, and truncation outside the 0 to $\frac{\pi}{2}$ rad interval. The azimuth angle $\omega$ follows a uniform distribution in $[0,2 \pi)$. Note that a circular cell radius $R=1.5 \mathrm{~m}$ is utilized in the multi-cell setting, which leads to a hexagonal cell apothem of approximately $\frac{\sqrt{3}}{2} 1.1 R \approx 0.953 R$.

Figure 3 represents the average cell data rate as a function of the inclination angle $\alpha$. As expected, the larger the inclination of the PD device, the lower is the average cell data rate as the higher is the probability of misalignment between the PD and the transmitting $\mathrm{AP}(\mathrm{s})$. Note that the cooperative transmission scheme offers a better performance than the non-cooperative scheme when the inclination angle is larger than a threshold value $\alpha_{\mathrm{th}} \approx 45^{\circ}$, where the misalignment probability with $\mathrm{AP}_{1}$ increases notably; due to that, only a cooperation strategy with the neighboring APs can maintain the communication in this situation. At $\alpha=0^{\circ}$, the cooperative scheme loses around $15 \%$ of the average cell data rate; however, when $\alpha \geq$ $65^{\circ}$, performance gains of more than $50 \%$ are observed. In addition, when $\alpha$ grows large, the non-cooperative scheme is in outage, whereas the cooperative scheme is able to provide a non-negative average cell data rate.

The average cell data rate as a function of the FoV is plotted in Fig. 4. The wider the FoV angle of the PD device, the larger is the average cell data rate as the higher is the probability of alignment with the serving AP. The maximum average cell data rate is achieved when the neighboring APs are within the FoV. Besides, there is a break point when $\Psi_{\mathrm{FoV}} \approx 20^{\circ}$, from which the interfering APs start to influence notably the level of co-channel interference that is present in the received signal. As expected, the cooperative transmission scheme provides better performance than the non-cooperative one at lower

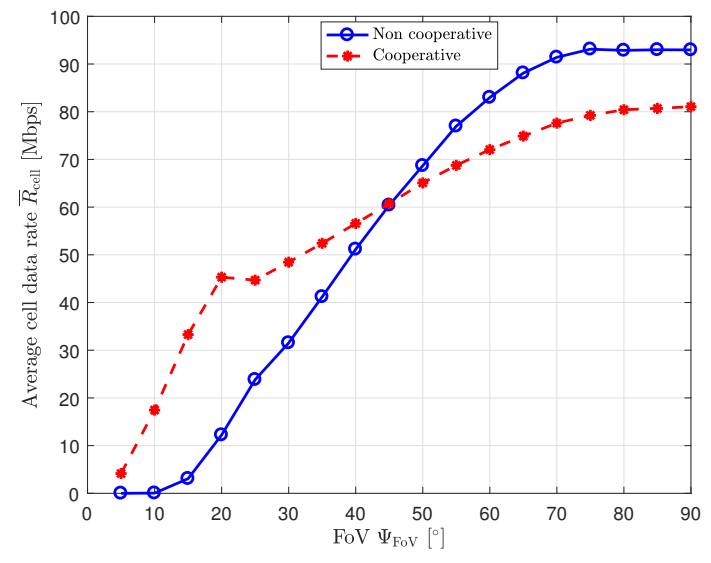

Fig. 4: Average cell data rate as a function of the FoV angle $\Psi_{\text {FoV }}$ for the (non-)cooperative transmission schemes with $\alpha=45^{\circ}$.

FoV angles, where the interference level is reduced and the performance gain comes from the multi-point transmission diversity strategy that the cooperative scheme provides.

From now on, a more realistic scenario is implemented, in which every PD is associated with a user (obstacle) that is placed in the same direction of the azimuth angle $\omega$ and at a distance $r_{\mathrm{user}-\mathrm{PD}}=0.3 \mathrm{~m}$. Thus, every user is located at coordinates $\left(r_{\text {user-PD }} \cdot \cos \omega, r_{\text {user-PD }} \cdot \sin \omega\right)$ and, thus, can block the LoS link between the PD and some of the APs. The shapes of the users (obstacles) are approximated by cylinders with a radius of $0.15 \mathrm{~m}$ and a height of $1.75 \mathrm{~m}$. To study the influence that the density of users (obstacles) has on the LoS-link blockage with every $\mathrm{AP}_{i}(i=1, \ldots, 19)$, the outage probability and the average cell data rate are shown in Fig. 5 and Fig. 6 when users are placed in the whole cell and the cell-edge areas, respectively. In Fig. 5a and Fig. 6a, it is possible to see that the larger is the index of the AP, the higher is the probability of LoS blockage. Fig. 5b and Fig. 6 b plot the outage probability computed analytically with (15), (16), and the LoS blockage probabilities reported in Fig. 5a and Fig. 6a, as well as the results obtained completely with numerical simulations. Note that the analytical results approximate better the simulation results when only celledge users are evaluated; this is because the $\mathrm{RVs} X_{i}$ are less correlated in this situation. In can also be observed that the cooperative scheme reduces considerably the outage probability with respect to the non-cooperative scheme. For example, when there is one obstacle per sector, a six-fold reduction is observed in the outage probability (this effect is reduced to a factor of two when the number of obstacles per sector grows to 4-5). When only the performance of the cell-edge users are evaluated (see Fig. 6), one notices that the cooperative transmission scheme guarantees a more homogeneous coverage, unlike the non-cooperative scheme in which cell-edge users suffer from a higher outage probability. In addition, when studying the results reported in Fig. 6c, it is possible to see that the average cell data rate when only celledge users are considered is much better in the cooperative scheme than in the non-cooperative one. When all the users are evaluated, as seen in Fig. 5c, both schemes offer similar performance. Note that, the higher is the inclination of the PD, the lower is the average cell data rate because the probability of having a LoS-link blockage towards the nearest AP is higher. 

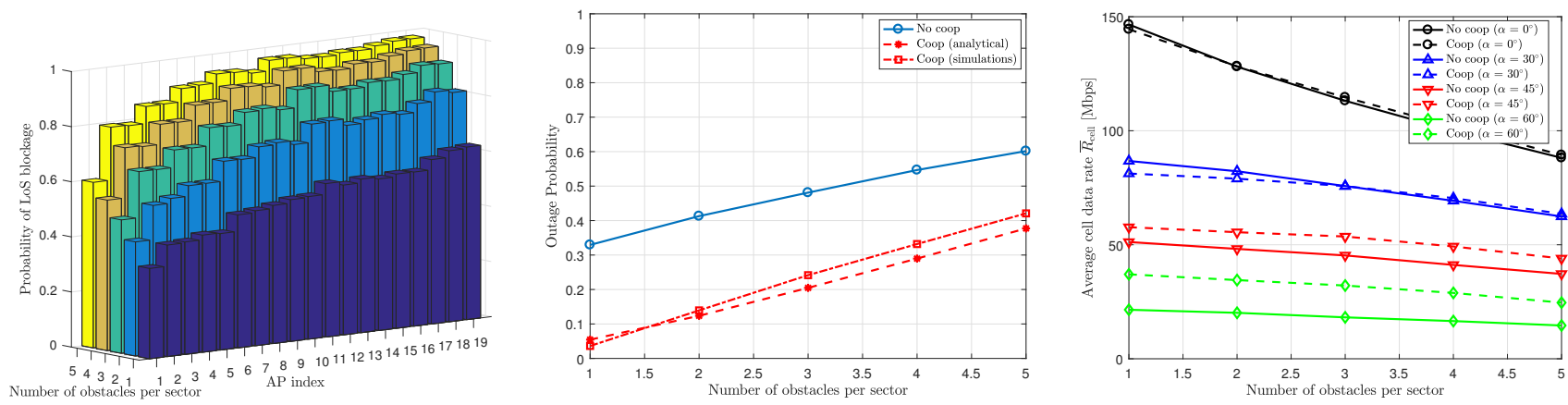

(a) Probability of Non-LoS between users (b) Outage probability as a function of the (c) Average cell data rate as a function of the located at the reference sector and every AP. number of users (obstacles) per sector. number of users (obstacles) per sector.

Fig. 5: Study of LoS blockage probability, outage probability and average cell data rate when users (obstacles) are located in the whole cell with $\Psi_{\mathrm{FoV}}=45^{\circ}$.
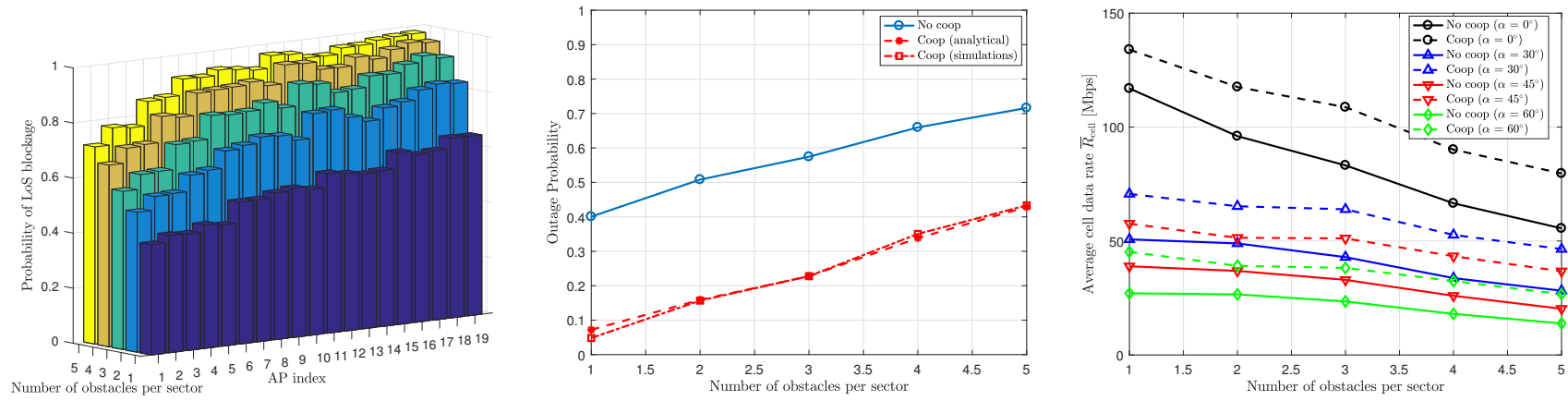

(a) Probability of Non-LoS between users (b) Outage probability as a function of the (c) Average cell data rate as a function of the located at the reference sector and every AP. number of users (obstacles) per sector. number of users (obstacles) per sector.

Fig. 6: Study of LoS blockage probability, outage probability and average cell data rate when users (obstacles) are located in the cell edge with $\Psi_{\mathrm{FoV}}=45^{\circ}$. The cell-edge area Area edge $_{\text {is defined as the outer ring area that verifies Area } \mathrm{edge}=0.1 \cdot \text { Area }}$ cell .

\section{CONCLUSION}

The use of a cooperative transmission scheme, as an enabler to address the misalignment and blockage events that impact the performance of a VLC system notably, has been considered in this paper. For this purpose, the effect of the orientation of the sensitive area of the PD, as well as the probability of existence of a LoS-link blockage event, have been evaluated using (non-)cooperative transmission in a VLC multi-cell scenario using RGB LEDs. Based on the obtained simulation results, it was possible to conclude that the inclination and FoV angles of the PD receiver affect the VLC system performance notably. It was also demonstrated that the use of a cooperative scheme offers a much more reliable and robust communication than the non-cooperative one, reducing the outage probability from six to two times when the number of obstacles per sector varied from one to five. Besides, important performance gains have been observed when evaluating the average cell data rate, particularly when users located in the cell-edge area of the VLC network were considered. In a practical setting, the proposed CoMP scheme can be easily implemented by using a centralized processing unit that manages the information to be transmitted by the different APs located in the same room.

\section{ACKNOWLEDGEMENTS}

This work has been partially funded by the Spanish MECD FPU fellowship program granted to the author B. Genovés Guzmán, the Catalan Government under Grant 2017SGR1479, and the Spanish Government under the national project 'TERESA-ADA' with ID no. TEC2017-90093C3-2-R/1-R (MINECO/AEI/FEDER, UE).

\section{REFERENCES}

[1] L. Hanzo, H. Haas, S. Imre, D. O’Brien, M. Rupp, and L. Gyongyosi, "Wireless myths, realities, and futures: from $3 \mathrm{G} / 4 \mathrm{G}$ to optical and quantum wireless," Proc. IEEE, vol. 100, no. Special Centennial Issue, pp. 1853-1888, May 2012.

[2] Z. Wang, Q. Wang, W. Huang, , and Z. Xu, Visible Light Communications: Modulation and Signal Processing. Wiley-IEEE Press, 2018

[3] 3GPP TR 36.814, V9.0.0, "Further advancements for EUTRA physical layer aspects," Mar. 2010.

[4] M. S. Demir, F. Miramirkhani, and M. Uysal, "Handover in VLC networks with coordinated multipoint transmission," in Proc. IEEE International Black Sea Conference on Communications and Networking, June 2017, pp. 1-5.

[5] C. Chen and H. Haas, "Performance evaluation of downlink cooperative multipoint joint transmission in LiFi systems," in Proc. IEEE Globecom Workshops, Dec. 2017, pp. 1-6.

[6] H. Haas, L. Yin, Y. Wang, and C. Chen, "What is LiFi?" J. Lightw. Technol., vol. 34, no. 6, pp. 1533-1544, Mar. 2016.

[7] B. G. Guzman, A. L. Serrano, and V. P. G. Jimenez, "Cooperative optical wireless transmission for improving performance in indoor scenarios for visible light communications," IEEE Trans. Consum. Electron., vol. 61, no. 4, pp. 393-401, Nov. 2015.

[8] B. G. Guzmán, A. A. Dowhuszko, V. P. G. Jiménez, and A. I. PérezNeira, "Robust cooperative multicarrier transmission scheme for optical wireless cellular networks," IEEE Photon. Technol. Lett., vol. 30, no. 2, pp. 197-200, Jan. 2018.

[9] D. Karunatilaka, V. Kalavally, and R. Parthiban, "Improving lighting quality and capacity of OFDM-based WDM-VLC systems," IEEE Photon. Technol. Lett., vol. 28, no. 20, pp. 2149-2152, Oct. 2016.

[10] M. D. Soltani, A. A. Purwita, Z. Zeng, H. Haas, and M. Safari, "Modeling the random orientation of mobile devices: measurement, analysis and LiFi use case," IEEE Trans. Commun., vol. 67, no. 3, pp. 2157-2172, Mar. 2019.

[11] M. Dehghani Soltani, A. A. Purwita, I. Tavakkolnia, H. Haas, and M. Safari, "Impact of device orientation on error performance of $\mathrm{LiFi}$ systems," IEEE Access, vol. 7, pp. 41 690-41 701, 2019.

[12] B. G. Guzmán, V. P. G. Jiménez, M. C. A. Torres, H. Haas, and L. Hanzo, "Downlink performance of optical OFDM in outdoor visible light communication," IEEE Access, vol. 6, pp. 76 854-76 866, 2018. 